ISSN 1855-3966 (printed edn.), ISSN 1855-3974 (electronic edn.)

ARS MATHEMATICA CONTEMPORANEA 20 (2021) 223-231

https://doi.org/10.26493/1855-3974.2173.71a

(Also available at http://amc-journal.eu)

\title{
Nordhaus-Gaddum type inequalities for the distinguishing index
}

\author{
Monika Pilśniak \\ AGH University, Department of Discrete Mathematics, \\ al. Mickiewicza 30, 30-059 Krakow, Poland
}

Received 6 November 2019, accepted 1 March 2021, published online 3 November 2021

\begin{abstract}
The distinguishing index of a graph $G$, denoted by $D^{\prime}(G)$, is the least number of colours in an edge colouring of $G$ not preserved by any nontrivial automorphism. This invariant is defined for any graph without $K_{2}$ as a connected component and without two isolated vertices, and such a graph is called admissible. We prove the Nordhaus-Gaddum type relation:

$$
2 \leq D^{\prime}(G)+D^{\prime}(\bar{G}) \leq \Delta(G)+2
$$

for every admissible connected graph $G$ of order $|G| \geq 7$ such that $\bar{G}$ is also admissible.

Keywords: Symmetry breaking in graphs, distinguishing index, Nordhaus-Gaddum type bounds.

Math. Subj. Class. (2020): 05C15, 05C25

\section{Introduction and main result}

We consider finite graphs and their edge colourings, not necessarily proper. Such a colouring breaks an automorphism of a graph if there exists an edge that is mapped into an edge with a different colour by that automorphism. A colouring is called asymmetric (or distinguishing), if it breaks all non-trivial automorphisms. The minimum number of colours in an asymmetric colouring of a graph $G$ is called the distinguishing index of $G$ and is denoted by $D^{\prime}(G)$. Obviously, the distinguishing index is defined only for graphs without $K_{2}$ as a component and with at most one isolated vertex. We call such graphs admissible.

The following general upper bound for the distinguishing index of connected graphs with respect to the maximum degree was proved by Kalinowski and Pilśniak in [8].
\end{abstract}

E-mail address: pilsniak@agh.edu.pl (Monika Pilśniak)

()(7) This work is licensed under https://creativecommons.org/licenses/by/4.0/ 
Theorem 1.1 ([8]). If $G$ is a connected graph with at least three vertices, then

$$
D^{\prime}(G) \leq \Delta(G)
$$

unless $G$ is $C_{3}, C_{4}$ or $C_{5}$.

This result was improved in [10], where all connected graphs with the distinguishing index equal to the maximum degree were characterized. In particular, a tree is called symmetric (respectively, bisymmetric) if it contains a central vertex $v_{0}$ (resp. a central edge $e_{0}$ ), all leaves are at the same distance from $v_{0}$ (resp. $\left.e_{0}\right)$ and all vertices that are not leaves have the same degree.

Theorem 1.2 ([10]). Let $G$ be a connected graph of order at least three. Then

$$
D^{\prime}(G) \leq \Delta(G)-1
$$

unless $G$ is a cycle, a symmetric or a bisymmetric tree, $K_{4}$ or $K_{3,3}$.

In the same paper, the conjecture for 2-connected graphs was formulated, and quite recently was confirmed in [7] in a bit stronger form, as follows.

Theorem 1.3 ([7]). If $G$ is a connected graph with minimum degree $\delta(G) \geq 2$, then

$$
D^{\prime}(G) \leq\lceil\sqrt{\Delta(G)}\rceil+1
$$

The main goal of the paper is a proof of a Nordhaus-Gaddum type inequalities for the distinguishing index of a graph. Our investigation was motivated by the renowned result of Nordhaus-Gaddum [9] who determined in 1956 lower and upper bounds for the sum of the chromatic numbers of a graph and its complement (actually, the upper bound was first proved by Zykov [12] in 1949). Since then, Nordhaus-Gaddum type bounds were obtained for many graph invariants. An exhaustive survey is given in [1]. In particular, it was considered by Collins and Trenk in [3] for the distinguishing chromatic number $\chi_{D}(G)$, which is the minimum number of colours in an asymmetric proper vertex colouring of a graph $G$. The Nordhaus-Gaddum type inequalities were also investigated in [10] for the chromatic distinguishing index $\chi_{D}^{\prime}(G)$ of a graph $G$ defined for asymmetric proper edge colourings. It was proved therein that if $G$ is an admissible graph of order $n \geq 3$ distinct from $K_{1,4}$, then

$$
n-1 \leq \chi_{D}^{\prime}(G)+\chi_{D}^{\prime}(\bar{G}) \leq 2(n-1) .
$$

Both upper and lower bounds are similar to Vizing bounds proved for the chromatic index of a graph [11] but in the proof for the chromatic distinguishing index we have to be more careful.

It was also conjectured in [10] that

$$
2 \leq D^{\prime}(G)+D^{\prime}(\bar{G}) \leq \max \{\Delta(G), \Delta(\bar{G})\}+2
$$

if both graphs $G$ and $\bar{G}$ are admissible and of order $n \geq 7$. It was confirmed for some classes of graphs, in particular for trees, claw-free graphs, 3-connected graphs and traceable graphs. Here, we prove the stronger version of Nordhaus-Gaddum type inequality for the distinguishing index. 
Theorem 1.4 (Main Theorem). If both $G$ and $\bar{G}$ are admissible graphs of order $n \geq 7$, and $G$ is connected, then

$$
2 \leq D^{\prime}(G)+D^{\prime}(\bar{G}) \leq \Delta(G)+2 .
$$

The lower bound by 2 is obvious. Indeed, if a graph $G$ is asymmetric, that means it has a trivial automorphism group, then the distinguishing index of both $G$ and $\bar{G}$ equals 1 . Moreover, Theorem 1.4 is tight. To see this, consider a symmetric (or bisymmetric) tree $T$ of order $n \geq 7$. Then by [5] $\bar{T}$ contains an asymmetric spanning tree if $T$ is different from a star, so $D^{\prime}(\bar{T})=2$ by Proposition 2.2. So, it follows from Theorem 1.2 that $D^{\prime}(T)+D^{\prime}(\bar{T})=\Delta(T)+2$ for symmetric and bisymmetric trees.

It has to be noted that there exist graphs of order less than 7 violating the right inequality in Theorem 1.4. For example, $D^{\prime}\left(K_{3,3}\right)=3, D^{\prime}\left(\overline{K_{3,3}}\right)=4$, whence $D^{\prime}\left(K_{3,3}\right)+$ $D^{\prime}\left(\overline{K_{3,3}}\right)=\Delta\left(K_{3,3}\right)+4$. Also, $D^{\prime}\left(C_{5}\right)+D^{\prime}\left(\overline{C_{5}}\right)=\Delta\left(C_{5}\right)+4$, and $D^{\prime}\left(K_{1, i}\right)+$ $D^{\prime}\left(\overline{K_{1, i}}\right)=\Delta\left(K_{1, i}\right)+3$ for $i=3,4,5$.

In Section 2 we recall some results useful in the proof of Theorem 1.4, which is given in Section 3.

\section{Known bounds for $D^{\prime}$}

Let us recall some useful results, before we start to prove the Main Theorem. A graph that contains a Hamiltonian path, i.e. a path that visits each vertex of the graph, is called traceable. Following [2], we define the $k$-th Bondy-Chvátal closure $\mathrm{cl}_{k}(G)$ of a graph $G$ as the graph obtained from $G$ by recursively joining pairs of non-adjacent vertices with degree-sum at least $k$. By the well-known theorem of Bondy and Chvátal [2], a graph $G$ of order $n$ is traceable whenever $\mathrm{cl}_{n-1}(G)$ is traceable.

We begin with the distinguishing index of complete graphs and of traceable graphs.

Proposition 2.1 ([8]).

$$
D^{\prime}\left(K_{n}\right)= \begin{cases}2, & \text { if } n \geq 6, \\ 3, & \text { if } n=3,4,5 .\end{cases}
$$

The following simple observation is very useful in some proofs. A subgraph $H$ of a graph $G$ is called almost spanning if $H$ is a spanning subgraph of a graph $G-v$ for some $v \in V(G)$.

Proposition 2.2 ([10]). If $H$ is a spanning or almost spanning subgraph with at least three vertices of a graph $G$, then $D^{\prime}(G) \leq D^{\prime}(H)+1$.

In particular, the spanning path in a traceable graph needs two colours to break its nontrivial automorphism, so a traceable graph has the distinguishing index at most 3. Actually we have a stronger result.

Theorem 2.3 ([10]). Let $G$ be a traceable graph of order at least 3 . If $G$ has at least 7 vertices, then $D^{\prime}(G) \leq 2$. Moreover, if $G$ is of order at most 6 , then $D^{\prime}(G) \leq 3$.

The distinguishing index of complete bipartite graphs was determined independently by Fisher and Isaak [4] and by Imrich, Jerebic and Klavžar [6]. Actually, they formulated their result for the distinguishing number $D\left(K_{p} \square K_{q}\right)$ of the Cartesian product of complete graphs, but $D^{\prime}\left(K_{p, q}\right)=D\left(K_{p} \square K_{q}\right)$. 
Theorem $2.4([4,6])$. Let $p, q$, $d$ be integers such that $d \geq 2$ and $(d-1)^{p}<q \leq d^{p}$. Then

$$
D^{\prime}\left(K_{p, q}\right)= \begin{cases}d, & \text { if } q \leq d^{p}-\left\lceil\log _{d} p\right\rceil-1 \\ d+1, & \text { if } q \geq d^{p}-\left\lceil\log _{d} p\right\rceil+1\end{cases}
$$

If $q=d^{p}-\left\lceil\log _{d} p\right\rceil$, then the distinguishing index $D^{\prime}\left(K_{p, q}\right)$ is either $d$ or $d+1$ and can be computed recursively in $O\left(\log ^{*}(q)\right)$ time.

In the rest of the paper, we make use of the following immediate corollary.

Corollary 2.5. If $p \leq q$, then $D^{\prime}\left(K_{p, q}\right) \leq\lceil\sqrt[p]{q}\rceil+1$.

The following simple observation is used later in this section.

Proposition 2.6. If $H$ is an admissible disconnected graph of order at least 7 , then $D^{\prime}(H) \leq|H|-2$.

Proof. Theorem 1.2 implies that the only connected graph $H$ with $D^{\prime}(H) \geq|H|-1$ is $K_{3}, K_{4}, C_{4}$ or a star $K_{1, n-1}$ for $n \geq 3$. If all components $H_{1}, \ldots, H_{s}$ of $H$ are pairwise non-isomorphic, then $D^{\prime}(H)=\max \left\{D^{\prime}\left(H_{i}\right): i=1, \ldots, s\right\}$, so $D^{\prime}(H) \leq|H|-2$. If $H$ contains $t \geq 2$ copies of a graph $H_{1}$ as its components, so $|H| \geq t\left|H_{1}\right|$, then we colour one of them distinguishingly and use one extra colour for each other copy. Hence,

$$
D^{\prime}\left(t H_{1}\right) \leq D^{\prime}\left(H_{1}\right)+t-1 \leq\left|H_{1}\right|+t-1 \leq \frac{|H|}{t}+t-1 \leq|H|-2 .
$$

We easily extend a result of [10] for trees to forests. First, recall a result of Hedetniemi et al. [5] on packing two trees into $K_{n}$.

Theorem 2.7 ([5]). A complete graph $K_{n}$ contains edge disjoint copies of any two trees of order $n$ distinct from a star $K_{1, n-1}$.

Proposition 2.8. Let $G$ be an admissible graph of order $n$ such that $\bar{G}$ is an admissible forest. Then $D^{\prime}(G) \leq 2$ if $n \geq 7$, and $D^{\prime}(G) \leq 3$ otherwise.

Proof. The case when $\bar{G}$ is a tree was proved in [10]. Otherwise, it easily follows from Theorem 2.7 that $G$ contains a Hamiltonian path $P_{n}$. Indeed, we can consider a tree $F^{\prime}$ spanned by $\bar{G}$, and every tree distinct from a star is included in a subgraph $\overline{F^{\prime}}$ of $G$. Thus $D^{\prime}(G) \leq 2$ if $n \geq 7$, and $D^{\prime}(G) \leq 3$ if $3 \leq n \leq 6$ by Theorem 2.3.

Additionally, let us note the following observation for small graphs. Denote by $W_{1}$ and $W_{2}$ the two graphs from Figure 1 called windmills.
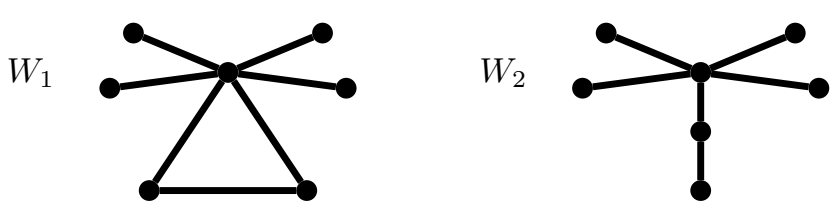

Figure 1: Two windmills. 
Proposition 2.9. If $G$ is a connected graph of order at most 7 different from windmills $W_{1}$ and $W_{2}$, and from a star $K_{1, n}$ for $n=4,5,6$, then $D^{\prime}(G) \leq 3$.

Proof. Observe that if $\Delta(G) \leq 4$, then the claim holds by Theorem 1.2. So let $\Delta(G) \geq 5$. First assume that $G$ does not have pendant edges. If the longest cycle in $G$ is of order at least 6 , then $G$ is traceable and $D^{\prime}(G) \leq 3$ by Theorem 2.3. If the longest cycle $C$ in $G$ is of order 5 , then we colour the edges of this cycle with $0,1,0,2,0$ (in this order) and all chords with 1 . Thus, each vertex of $C$ has an incident edge coloured with 0 . If there exists a vertex of $G$ with noncoloured incident edges, then all of them we colour with 2 and all the remaining edges with 1 . It is an asymmetric colouring of $G$, because outside the initial cycle $C$ we create a monochromatic vertex with colour 2 and a monochromatic vertex with colour 1 or a bichromatic one with colours 1 and 2 .

If the longest cycle in $G$ is of order 4 , then a 2-connected $G$ is isomorphic to $K_{2, r}$ or $K_{2, r}+e$ with $r \leq 5$ where $e$ is an edge between two vertices of maximum degree. Three colour suffice for an asymmetric colouring of $r$ paths of length two between the two vertices of maximum degree. If such a 2-connected graph is joined with a triangle (in a cut vertex), then we can colour every edge of the triangle with a different colour. If two cycle of length 4 (with chords) meet in only one vertex, then an asymmetric colouring of one of them uses colour 1 twice, while the other one uses 2 twice (apart from one edge coloured with 0$)$.

If the longest cycle is a triangle $C_{3}$, then $G$ has a cut vertex $v$ where three cycles meet. Then a pair of edges including $v$ in every triangle is coloured with 1 and 2 , while every remaining edge obtains a distinct colour 1,2 or 3 .

Now assume that there exists a leaf $v$ in $G$. First suppose there exists an induced subgraph $B$ of $G$ with minimal degree at least 2 . Then we can colour it with three colours as above. So we have to distinguish now only pendant trees (in particular paths and edges) with a common vertex just fixed in $B$. It is always possible to do this with three colours if $|G| \leq 7$ unless $B$ has a pendant star $K_{1,4}$. Then we obtain an exceptional graph $W_{1}$.

Finally, let $G$ be a tree of order at most 7 different from a star $K_{1, n}$ with $n=3, \ldots, 6$. Then $D^{\prime}(G) \leq \Delta(G)-1$ by Theorem 1.2. Clearly, $D^{\prime}(G) \leq 3$ unless $G=W_{2}$, which needs four colours like $W_{1}$.

\section{Proof of the Main Theorem}

Proof of Theorem 1.4. Let $G$ be a connected graph of order $n \geq 7$ such that both $G$ and its complement $\bar{G}$ are admissible. Denote $\Delta=\Delta(G)$. Clearly, $\Delta \geq \Delta(\bar{G})+1$ whenever $\bar{G}$ is disconnected. Next, if $\Delta \leq \frac{n-1}{2}$, then

$$
\delta(\bar{G})=n-1-\Delta \geq \frac{n-1}{2},
$$

and $\bar{G}$ is connected. Otherwise, $\frac{n-1}{2} \geq \Delta \geq \Delta(\bar{G})+1 \geq \frac{n-1}{2}+1$. Hence, $\bar{G}$ satisfies the well-known Dirac's condition for traceability, so $\bar{G}$ is traceable. Hence, $D^{\prime}(G)+$ $D^{\prime}(\bar{G}) \leq \Delta+2$ by Theorem 1.1 and Theorem 2.3 .

Assume then that $\Delta \geq\left\lceil\frac{n}{2}\right\rceil$. Analogously, we can assume that $\Delta(\bar{G}) \geq\left\lceil\frac{n}{2}\right\rceil$. Indeed, our theorem holds if $\Delta(\bar{G}) \leq \frac{n-1}{2}$. Then $D^{\prime}(G) \leq 2$ and $D^{\prime}(\bar{G}) \leq \Delta(\bar{G}) \leq \frac{n-1}{2} \leq \Delta$ if $\bar{G}$ is connected, or $D^{\prime}(\bar{G}) \leq\left\lceil\frac{n}{2}\right\rceil \leq \Delta$ if $\bar{G}$ is disconnected, by the following reasons.

Theorem 1.1 implies that the only connected graphs $H$ with $D^{\prime}(H)>\Delta(H)$ are $C_{3}, C_{4}$ and $C_{5}$. If all components $H_{1}, \ldots, H_{s}$ of $\bar{G}$ are pairwise non-isomorphic, then 
$D^{\prime}(\bar{G})=\max \left\{D^{\prime}\left(H_{i}\right): i=1, \ldots, s\right\} \leq \max \left\{3, \Delta\left(H_{i}\right)\right\}$, so $D^{\prime}(\bar{G}) \leq \frac{n}{2}$. If $\bar{G}$ contains $t_{i} \geq 2$ copies of a graph $H_{i}$ as its components, then let $t=\max \left\{t_{i}: i=1, \ldots, s\right\}$ and let $H=H_{l}$ where $D^{\prime}\left(H_{l}\right)=\max \left\{D^{\prime}\left(H_{i}\right): i=1, \ldots, s\right\}$, and we colour one copy of $H_{l}$ distinguishingly and use one extra colour for each other copy. So, for $H$ different from $C_{3}$, $C_{4}$ and $C_{5}$ we have

$$
D^{\prime}(\bar{G}) \leq D^{\prime}\left(t H_{l}\right) \leq D^{\prime}\left(H_{l}\right)+t-1 \leq \Delta\left(H_{l}\right)+t-1 \leq \frac{|\bar{G}|}{t}+t-2 \leq \frac{n}{2},
$$

and for $i \in\{3,4,5\}$ we obtain $D^{\prime}\left(t C_{i}\right) \leq t+2 \leq\left\lceil\frac{t i}{2}\right\rceil$.

We distinguish two cases.

Case A: Both $G$ and $\bar{G}$ are connected graphs without pendant edges.

Then $D^{\prime}(G) \leq\lceil\sqrt{\Delta}\rceil+1$ and $D^{\prime}(\bar{G}) \leq\lceil\sqrt{\Delta(\bar{G})}\rceil+1$. Hence, the inequality $D^{\prime}(G)+$ $D^{\prime}(\bar{G}) \leq \Delta+2$ is weaker than

$$
\Delta-\lceil\sqrt{\Delta}\rceil \geq\lceil\sqrt{\Delta(\bar{G})}\rceil .
$$

First assume that $\Delta \geq \Delta(\bar{G})$. It is easy to see that the inequality (3.1) is satisfied unless $\Delta=\Delta(\bar{G})=5$. In the latter case $8 \leq n \leq 10$ and $\delta(G)=\delta(\bar{G})=n-6$. We want to show that either $G$ or its complement $\bar{G}$ is traceable.

We say that a sequence $\left(a_{i}\right)$ is minorized by a sequence $\left(b_{i}\right)$ if $b_{i} \leq a_{i}$ for any $i$. If $n=8$, then the degree sequence, ordered non-increasingly, of $G$ (or $\bar{G}$ ) is minorized by $(5,5,4,4,2,2,2,2)$ or by $(5,4,4,4,3,2,2,2)$. Indeed, we know by assumptions that $b_{1}=5, b_{8}=2$, two terms of $b_{i}$ have to be odd since the sum of degrees is even in every graph, and the sum of the fourth term of the sequence of $G$ and the fifth term of the sequence of $\bar{G}$ equals $n-1=7$, so one of them cannot be smaller than $\left\lceil\frac{n-1}{2}\right\rceil=4$. Now, by definition of the $(n-1)$-th Bondy-Chvátal closure $\mathrm{cl}_{n-1}(G)$, a vertex of degree five in $G$ has degree $n-1=7$ in $\operatorname{cl}_{7}(G)$, so we have to add two new edges incident to it. Observe that adding new edges yields another vertex that has degree $n-1$ in $\operatorname{cl}_{7}(G)$, and this is the case at each step of creating $\mathrm{cl}_{7}(G)$. Finally, $\operatorname{cl}_{7}(G)=K_{8}$. Hence, $G$ is traceable, by the Bondy-Chvátal theorem [2].

Similarly, if $n=9$, we may assume that the degree sequence of $G$ is minorized by $(5,4,4,4,4,4,3,3,3)$ or by $(5,5,4,4,4,3,3,3,3)$, and it is not difficult to see that $\mathrm{cl}_{8}(G)=K_{9}$. For $n=10$, the degree sequence of $G$ is minorized by $(5,5,4, \ldots, 4)$ and here it is clear that $\operatorname{cl}_{9}(G)=K_{10}$. For brevity, the details are left to the reader.

Now assume that $\Delta(\bar{G})>\Delta$. Then it is easily seen that the inequality (3.1) holds for any $\Delta, \Delta(\bar{G})$ and $n$ unless either $n=8, \Delta=4, \Delta(\bar{G})=5$, or $n=9, \Delta=5, \Delta(\bar{G})=6$, or $n=10, \Delta=5, \Delta(\bar{G}) \in\{6,7\}$, since $\left\lceil\frac{n}{2}\right\rceil-\left\lceil\sqrt{\left.\left\lceil\frac{n}{2}\right\rceil\right\rceil}\lceil\lceil\sqrt{n-3}\rceil\right.$. The same argument as above confirms that $\bar{G}$ is traceable.

Case B: A graph $\bar{G}$ is disconnected or $\delta(\bar{G})=1$.

If $\bar{G}$ is a forest, then the conclusion follows from Proposition 2.8. Hence, assume that $\bar{G}$ contains a 2 -connected block. We now consider decompositions of $\bar{G}$ into two subgraphs $F_{1}, F_{2}$ such that $E\left(F_{1}\right) \cup E\left(F_{2}\right)=E(\bar{G})$ and the vertex sets $V\left(F_{1}\right), V\left(F_{2}\right)$ share at most one vertex which is a cut-vertex of $\bar{G}$. Denote $p=\left|F_{1}\right|-1, q=\left|F_{2}\right|-1$ if $F_{1}$ and $F_{2}$ share a common vertex, and $p=\left|F_{1}\right|, q=\left|F_{2}\right|$ if $F_{1}$ and $F_{2}$ are disjoint. Assume that $p \leq q$ and the difference $q-p$ is smallest possible. Observe that $\Delta(G) \geq q$ and $G$ is spanned or almost spanned by a complete bipartite graph $K_{p, q}$. 
First, suppose that $q \leq 2^{p}-p$. Then $D^{\prime}(G) \leq 2$, since $G$ is (almost) spanned by an asymmetric spanning subgraph of $K_{p, q}$ for $p+q \geq 7$ by Proposition 3.10 in [10] (see also [6]).

If $\bar{G}$ is connected, then $\Delta=\Delta(G)=n-2$ since $\delta(\bar{G})=1$ by the assumption of Case B. Moreover, $D^{\prime}(\bar{G}) \leq n-2$ by Theorem 1.1 as $\Delta(\bar{G}) \leq n-2$. Hence

$$
D^{\prime}(G)+D^{\prime}(\bar{G}) \leq 2+n-2=\Delta+2 .
$$

If $\bar{G}$ is disconnected, then either $\delta(\bar{G})=1$ or $\delta(\bar{G}) \geq 2$. If $\delta(\bar{G})=1$, then $D^{\prime}(\bar{G}) \leq$ $n-2$ by Proposition 2.6 and $D^{\prime}(G)+D^{\prime}(\bar{G}) \leq 2+n-2=\Delta+2$. Now, let $\delta(\bar{G}) \geq 2$, and assume (in the worst case) that $\bar{G}$ contains $s$ components isomorphic to a connected graph $H$. Recall that $\Delta(\bar{G}) \geq \frac{n}{2}$, as we assumed at the beginning of the proof. So, $3 \leq|H|<\frac{n}{2 s}$. If we use a distinct additional colour for every component $H$, then $D^{\prime}(\bar{G}) \leq$ $\lceil\sqrt{\Delta(\bar{G})}\rceil+s \leq\lceil\sqrt{n-2}\rceil+s$, by Theorem 1.3. So, we would like to show that

$$
\lceil\sqrt{n-2}\rceil+s+2 \leq n-|H|+2,
$$

since $\Delta(G) \geq n-|H|$. To confirm the inequality (3.2), we estimate

$$
\lceil\sqrt{n-2}\rceil+s \leq\lceil\sqrt{n-2}\rceil+\frac{n}{6} \leq n-\frac{n}{4} \leq n-|H| .
$$

It is easy to verify that the second inequality is always true. The last inequality is obvious if $s$ is at least 2 . If $s=1$ we do not need to distinguishing connected component one from another, hence we use the same colours in every component and $D^{\prime}(\bar{G}) \leq\lceil\sqrt{\Delta(\bar{G})}\rceil+1 \leq$ $\Delta$. So, this subcase is completed.

Now, assume that $q \geq 2^{p}-p+1$. Then $D^{\prime}(G) \leq \sqrt[p]{q}+2$ for $p \geq 2$ by Corollary 2.5 and Proposition 2.2. In this case the graph $\bar{G}$ can be obtained from a 2-connected graph $B$ by attaching a number of independent pendant subgraphs (of order at most $p$ ) to it or there is a component of order $p$ and a 2-connected component $B$ of order $q$. Hence, $\Delta(B) \leq q$ and $D^{\prime}(\bar{G}) \leq\lceil\sqrt{q}\rceil+1$, by Theorem 1.3 for the block $B$, and by the observation that then every subgraph attached to $B$ has one vertex fixed and the order at most $p \leq \sqrt{q}+2$. We obtain $D^{\prime}(G)+D^{\prime}(\bar{G}) \leq\lceil\sqrt[p]{q}\rceil+2+\lceil\sqrt{q}\rceil+1$. Recall that $\Delta \geq q$, so it is enough to check whether

$$
\lceil\sqrt[p]{q}\rceil+\lceil\sqrt{q}\rceil+3 \leq q+2 .
$$

Consequently, we obtain the inequality

$$
q-\lceil\sqrt[p]{q}\rceil-\lceil\sqrt{q}\rceil-1 \geq 0
$$

For $p=3$, we have $q \geq 2^{3}-3+1=6$. For $q=6, p=3$ we have $D^{\prime}(G) \leq 4$ by Proposition 2.2 , and $D^{\prime}(\bar{G}) \leq 3$ by Proposition 2.9 for $F_{2}$. Observe now that the inequality (3.3) is satisfied, because it holds for $q=7$, and $q-\lceil\sqrt[3]{q}\rceil-\lceil\sqrt{q}\rceil-1$ is an increasing function of $q$. The inequality (3.3) also holds for larger values of $p$ since its left-hand side is non-decreasing with respect to $p$.

If $p=2$, then inequality (3.3) is satisfied for $q \geq 7$. For $q=5$ or $q=6, G$ is (almost) spanned by $K_{2,5}$ or $K_{2,6}$, so $D^{\prime}(G) \leq 4$ by Proposition 2.2 , and $D^{\prime}(\bar{G}) \leq 3$ by Proposition 2.9 for $F_{2}$. For $q=4$ we have $n=7$ and our theorem is true by Proposition 2.9.

Now let $p=1$. If $\bar{G}$ is disconnected, then $\bar{G}$ contains a 2 -connected block $B$ of order $n-1$ and an isolated vertex $v$. Hence, $\Delta=n-1$, of course. Then $D^{\prime}(\bar{G}) \leq D^{\prime}(B) \leq$ 
$\lceil\sqrt{\Delta(\bar{G})}\rceil+1$. Moreover, $D^{\prime}(G) \leq\lceil\sqrt{\Delta}\rceil+1$ whenever $\delta(G) \geq 2$, by Theorem 1.3. Hence,

$$
D^{\prime}(G)+D^{\prime}(\bar{G}) \leq 2\lceil\sqrt{\Delta}\rceil+2,
$$

and $2\lceil\sqrt{\Delta}\rceil+2 \leq \Delta+2$ for $\Delta \geq 6$, since $\Delta=n-1 \geq 6$.

If the graph $\overline{\bar{G}}$ is connected, then $\bar{G}$ can be obtained from a 2-connected block $B$ by attaching a number (maybe zero) of independent pendant edges to it. It is enough to break all nontrivial automorphisms of $B$. Then $D^{\prime}(\bar{G}) \leq D^{\prime}(B) \leq\lceil\sqrt{\Delta(\bar{G})}\rceil+1$. Moreover, $\Delta=n-2$ and $D^{\prime}(G) \leq\lceil\sqrt{\Delta}\rceil+1$ whenever $\delta(G) \geq 2$, by Theorem 1.3. Then $2\lceil\sqrt{\Delta}\rceil+$ $2 \leq \Delta+2$ for $\Delta \geq 6$, unless $\Delta=5$. But in the latter case $n=7$ and $D^{\prime}(G)+D^{\prime}(\bar{G}) \leq 6$ by Proposition 2.9.

Finally, assume that $p=1$ and $\delta(G)=1$. Then we consider decompositions of $G$ into two subgraphs $F_{1}^{\prime}, F_{2}^{\prime}$ such that $E\left(F_{1}^{\prime}\right) \cup E\left(F_{2}^{\prime}\right)=E(G)$ and the vertex sets $V\left(F_{1}^{\prime}\right), V\left(F_{2}^{\prime}\right)$ share one vertex which is a cut-vertex of $G$. Denote $p^{\prime}=\left|F_{1}^{\prime}\right|-1, q^{\prime}=\left|F_{2}^{\prime}\right|-1$. Assume that $p^{\prime} \leq q^{\prime}$ and the difference $q^{\prime}-p^{\prime}$ is smallest possible. Recall that $\Delta(\bar{G})=n-2$ and $\bar{G}$ is spanned or almost spanned by a complete bipartite graph $K_{p^{\prime}, q^{\prime}}$.

If $q^{\prime} \leq 2^{p^{\prime}}-p^{\prime}$, then $D^{\prime}(\bar{G}) \leq 2$ like above (for $p^{\prime}+q^{\prime} \geq 7$ ), and $D^{\prime}(G) \leq \Delta$ by Theorem 1.1. So, we are done. If $q^{\prime} \geq 2^{p^{\prime}}-p^{\prime}+1$, then $D^{\prime}(\overline{\bar{G}}) \leq \sqrt[p^{\prime}]{q^{\prime}}+2$ for $p^{\prime} \geq 2$, and we obtain

$$
D^{\prime}(G)+D^{\prime}(\bar{G}) \leq\left\lceil\sqrt{q^{\prime}}\right\rceil+\left\lceil\sqrt[p^{\prime}]{q^{\prime}}\right\rceil+3 \leq p^{\prime}+q^{\prime}+1
$$

since $\Delta \geq n-2=p^{\prime}+q^{\prime}-1$. For $p^{\prime}=2$, we have $q^{\prime} \geq 2^{2}-2+1=3$, and the inequality $2\left\lceil\sqrt{q^{\prime}}\right\rceil \leq q^{\prime}$ is satisfied since $n \geq 7$. Hence $q^{\prime} \geq 4$. The inequality $p^{\prime}+q^{\prime}-\left\lceil\sqrt{q^{\prime}}\right\rceil-\left\lceil\sqrt[p^{\prime}]{q^{\prime}}\right\rceil-2 \geq 0$ also holds for larger values of $p^{\prime}$ since its left-hand side is non-decreasing with respect to $p^{\prime}$.

Let $p^{\prime}=1$. Then there exists a 2 -connected block $B^{\prime}$ in $G$ with a number of independent pendant edges attached to it (at least one). Hence $D^{\prime}(G) \leq D^{\prime}\left(B^{\prime}\right) \leq\lceil\sqrt{\Delta}\rceil+1$. Recall that also $D^{\prime}(\bar{G}) \leq\lceil\sqrt{\Delta(\bar{G})}\rceil+1 \leq\lceil\sqrt{\Delta}\rceil+1$, since $\Delta=\Delta(\bar{G})=n-2$. So we verify the following inequality

$$
2\lceil\sqrt{\Delta}\rceil+2 \leq \Delta+2
$$

for $\Delta \in\{n-2, n-1\}$, which is true for $n \geq 7$ unless $\Delta=5$. But then $n=7$ and $D^{\prime}(G)+D^{\prime}(\bar{G}) \leq 6$ once more by Proposition 2.9 .

\section{ORCID iD}

Monika Pilśniak (D) https://orcid.org/0000-0002-3734-7230

\section{References}

[1] M. Aouchiche and P. Hansen, A survey of Nordhaus-Gaddum type relations, Discrete Appl. Math. 161 (2013), 466-546, doi:10.1016/j.dam.2011.12.018.

[2] J. A. Bondy and V. Chvátal, A method in graph theory, Discrete Math. 15 (1976), 111-135, doi:10.1016/0012-365x(76)90078-9.

[3] K. L. Collins and A. N. Trenk, Nordhaus-Gaddum theorem for the distinguishing chromatic number, Electron. J. Combin. 20 (2013), \#P46 (18 pages), doi:10.37236/2117. 
[4] M. J. Fisher and G. Isaak, Distinguishing colorings of Cartesian products of complete graphs, Discrete Math. 308 (2008), 2240-2246, doi:10.1016/j.disc.2007.04.070.

[5] S. M. Hedetniemi, S. T. Hedetniemi and P. J. Slater, A note on packing two trees into $K_{N}$, Ars Combin. 11 (1981), 149-153.

[6] W. Imrich, J. Jerebic and S. Klavžar, The distinguishing number of Cartesian products of complete graphs, European J. Combin. 29 (2008), 922-929, doi:10.1016/j.ejc.2007.11.018.

[7] W. Imrich, R. Kalinowski, M. Pilśniak and M. Woźniak, The distinguishing index of connected graphs without pendant edges, Ars Math. Contemp. 18 (2020), 117-126, doi:10.26493/ 1855-3974.1852.4f7.

[8] R. Kalinowski and M. Pilśniak, Distinguishing graphs by edge-colourings, European J. Combin. 45 (2015), 124-131, doi:10.1016/j.ejc.2014.11.003.

[9] E. A. Nordhaus and J. W. Gaddum, On complementary graphs, Amer. Math. Monthly 63 (1956), 175-177, doi:10.2307/2306658.

[10] M. Pilśniak, Improving upper bounds for the distinguishing index, Ars Math. Contemp. 13 (2017), 259-274, doi:10.26493/1855-3974.981.ff0.

[11] V. G. Vizing, The chromatic class of multigraphs, Kibernetika (Kiev) 1 (1965), 29-39.

[12] A. A. Zykov, On some properties of linear complexes, Mat. Sb. (N.S.) 24 (1949), 163-188, http://mi.mathnet.ru/eng/msb5974. 